\title{
Young Japanese College Students with Dysmenorrhea Have High Frequency of Irregular Menstruation and Premenstrual Symptoms
}

\author{
Tomoko Fujiwara ${ }^{*}, 1$ and Rieko Nakata ${ }^{2}$ \\ ${ }^{1}$ Faculty of Home Economics, Ashiya College, Ashiya, ${ }^{2}$ Department of Food Science and Nutrition, Nara Women's Uni- \\ versity, Nara, Japan
}

\begin{abstract}
In this study, to estimate the current status of young women with menstrual disorders, the relation among dysmenorrhea, irregular menstruation and premenstrual symptoms was investigated by a questionnaire. Subjects ranging from 18 to 20 years old were recruited from 522 female students at Ashiya College in Japan. The intensity of dysmenorrhea was classified into 3 grades (score 1, not requiring analgesic; score 2, painful, requiring analgesic; score 3, painful, not relieved by analgesic). All participants were further divided into subsequent groups as having premenstrual symptoms or not and those having regular or irregular menstruation. Dysmenorrhea scores in the students with premenstrual symptoms or irregular menstruation were significantly higher than those without these symptoms $(1.66 \pm 0.66 v s 1.41 \pm 0.59 ; 1.62 \pm 0.68$ vs $1.49 \pm 0.61$, respectively). There was no significant relation in the incidence between premenstrual symptoms and irregular menstruation. These findings suggest that considerable numbers of young women with dysmenorrhea are associated with premenstrual symptoms.
\end{abstract}

Keywords: Dysmenorrhea, irregular menses, premenstrual symptom, young women.

\section{INTRODUCTION}

Menstrual disorders, especially dysmenorrhea and premenstrual syndrome, are considered to affect the quality of life (QOL) of women during reproductive age. When the symptoms are severe, social activity is very limited during menstruation, reducing QOL in women. These disorders are frequently encountered in young women around adolescence [1]. Menstrual and reproductive factors including estrogenic agents have also been proposed to be associated with malignant diseases in the reproductive organs such as endometrial carcinoma and breast carcinoma as well as those in other organs [2,3]. From these perspectives, it is important to precisely evaluate the current status of menstrual disorders in young women in order to manage not only the present, but also future QOL.

Dysmenorrhea is mainly classified into functional dysmenorrhea and organic dysmenorrhea based on pathogenesis [1]. In general, functional dysmenorrhea is caused by ovarian dysfunction, which is frequently manifested as irregular menstruation. Since this disorder is commonly derived from immaturity of hypothalamic-pituitary-ovarian function, the symptom can be expected to spontaneously recover along with the maturation process of endocrinological feedback system. In contrast, endometriosis and uterine myoma, which are representative diseases causing organic dysmenorrhea, gradually become extended along with sequential menstruations, inducing constant pelvic pain throughout the menstrual cycle [4]. During reproductive ages, since these diseases may cause infertility in the future. evaluation of the current status of menstrual disorders in young women should be an important social issue [5].

*Address correspondence to this author at the Faculty of Home Economics, Ashiya College, 14-10 Rokurokuso-cho, Ashiya, Japan 659-8511; Tel: 81797-23-0663; Fax: 81-797-38-6705; E-mail: tomokof@bd5.so-net.ne.jp
On the other hand, premenstrual syndrome is a multifactorial syndrome that affects adolescent girls with a high frequency. This syndrome is characterized by manifestation of pschychological disorders such as irritability, depression, and mood swings and/or somatic complaints including abdominal bloating, peripheral edema, general fatigue and acne before menstruation [6]. These symptoms are recurrent according to menstrual cycles and rapidly disappeared just after onset of menstruation. Although various etiologies of premenstrual syndrome such as elevated prolactin levels, hypoglycemia or vitamin deficiencies have been proposed, none of these theories has been definitively proven [7].

In this study, to estimate the current status of young women with menstrual disorders in Japan, the relation between dysmenorrhea and premenstrual symptoms as well as irregular menstruation was investigated by responses to a questionnaire from female students ranging from 18 to 20 years old, who studied at Ashiya College during the recent 5 years.

\section{MATERIALS AND METHODOLOGY}

\section{Study Population}

Subjects were recruited from young Japanese women aged from 18 to 20 years old who studied at Ashiya College. The study protocol was approved by the Committee on Food Culture at Ashiya College. We sent questionnaires to all students who belong to faculty of home economics and child education between 2001 and $2005(\mathrm{n}=553)$. Information regarding the aim of this study was sent with the questionnaire and consent was obtained from all participants.

\section{Data Collection About Dysmenorrhea, Irregular Men- struation and Premenstrual Symptoms}

The intensity of dysmenorrhea was scored using 3 grades as previously described [8], consisting of grade 1 (free of 
pain or painful, but can manage without analgesic; score 1), grade 2 (painful, requiring analgesic; score 2), and grade 3 (painful, not relieved by analgesic; score 3 ).

In addition, all participants were divided into 2 groups as having regular or irregular menstruation. Regular menstruation is strictly defined as constant 26-32-day intervals in each menstrual cycle.

The participants were further divided into 2 groups in relation to the presence or absence of premenstrual symptoms. The students were requested to answer their experiences of 10 symptoms and signs during the premenstrual phases: pschychological disorders such as irritability, depression, nervousness and mood swings and/or somatic complaints such as abdominal bloating, peripheral edema, lumbago, headache, general fatigue and acne [6]. In this study, when the students experienced one or more of the above symptoms that rapidly disappeared just after onset of menstruation for at least three or more sequential menstrual cycles, they were classified as having premenstrual symptoms.

\section{Body Mass Index (BMI) Assessment}

Information on body mass (kilograms) and stature (meters) were obtained from all participants. Data on body mass and stature were based on physical examination organized by the Health Center at Ashiya College. Body mass index (BMI) was calculated using the formula: body weight in kilograms divided by height in meters squared.

\section{Statistical Analysis}

The data are shown as means \pm standard deviation (SD). Differences in the intensity of dysmenorrhea and BMI in the groups with or without irregular menstruation or premenstrual symptoms were analyzed by unpaired $t$ test. Differences in the incidence of irregular menstruation and premenstrual symptoms were analyzed by chi-square test. A $P$-value less than 0.05 was considered significant.

\section{RESULTS}

\section{The Relation Between Scores for Dysmenorrhea and Ir- regular Menstruation or Premenstrual Symptoms}

We obtained complete responses that were suitable for statistical analysis from 522 female students, who belong to faculty of home economics and child education between 2001 and 2005.

Among these, 174 students (33.3\%) reported irregular menstruation and 348 students (66.7\%) reported regular menses. Dysmenorrhea scores in students with irregular menstruation were significantly higher than those in students with regular menstruation ( $1.62 \pm 0.68$ vs $1.49 \pm 0.61, \mathrm{P}<0.05$, Fig. (1)).

On the other hand, 252 students $(48.3 \%)$ had premenstrual symptoms and 270 students $(51.7 \%)$ did not. Dysmenorrhea scores in students with premenstrual symptoms were significantly higher than those without them $(1.66 \pm 0.66 v s$ $1.41 \pm 0.59, \mathrm{P}<0.01$, Fig. (2)).

The same tendencies for both relations were observed in each year throughout the examined period between 2001 and 2005.

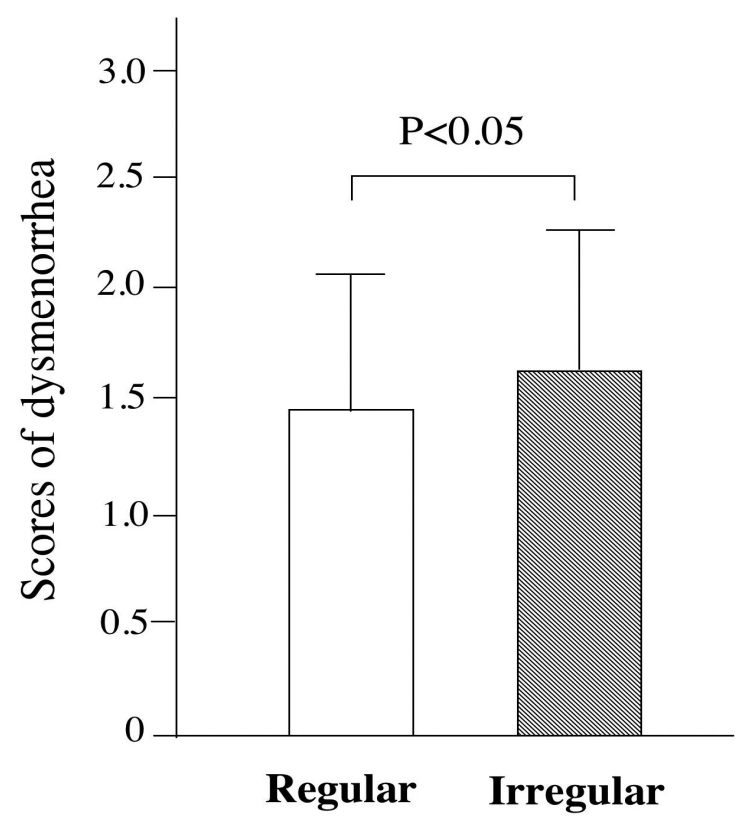

Fig. (1). Scores for dysmenorrhea in the students with regular and irregular menstruation. Dysmenorrhea scores in the students $(\mathrm{n}=174,33.3 \%)$ with irregular menstruation were significantly higher than those $(\mathrm{n}=348,66.7 \%)$ with regular menstruation $(P<0.05)$. The error bars show standard deviation.

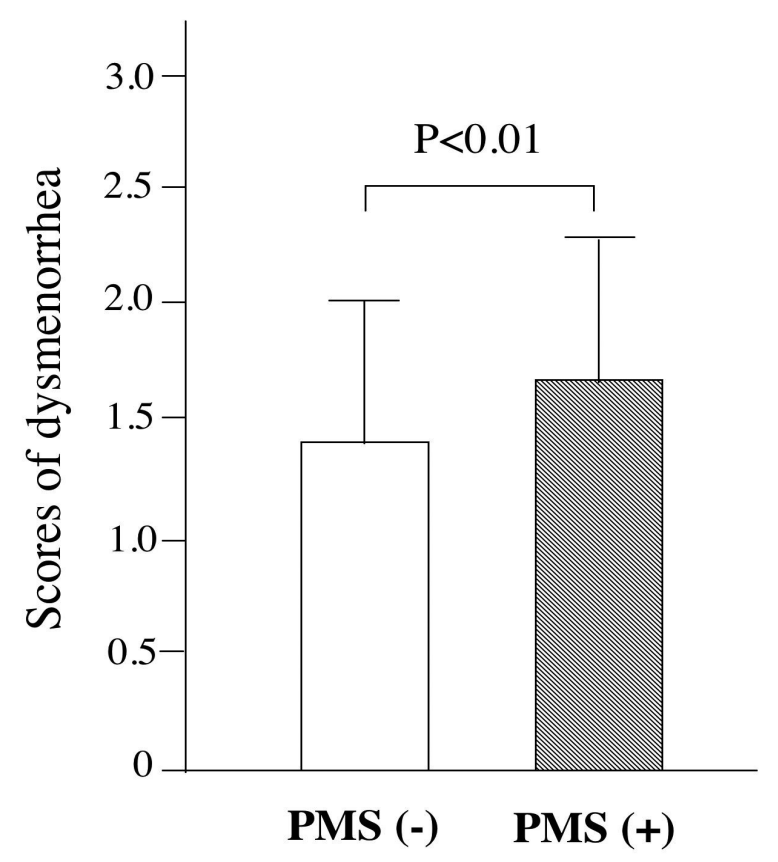

Fig. (2). Scores for dysmenorrhea in the students with or without premenstrual symptoms. Dysmenorrhea scores in the students $(\mathrm{n}=252,48.3 \%)$ with premenstrual symptoms (PMS) were significantly higher than those $(\mathrm{n}=270,51.7 \%)$ without symptoms $(P<0.01)$. The error bars show standard deviation.

\section{The Relation Between Irregular Menstruation and Pre- menstrual Symptoms}

There were no significant relation between the incidences of premenstrual symptoms and irregular menstruation (Table 1). 
Table 1. The Incidences of Premenstrual Symptoms and Irregular Menstruation

\begin{tabular}{|cccc|}
\hline & & \multicolumn{2}{c|}{ Irregular Menstruation } \\
& Positive & Negative \\
\hline \hline Premenstrual symptoms & & & \\
& Positive & 77 & 175 \\
& Negative & 97 & 173 \\
\hline
\end{tabular}

There were no significant relation between the incidences of premenstrual symptoms and irregular menstruation $(\mathrm{P}=0.159)$.

\section{The Differences in BMI Scores}

Students with irregular menstruation showed slightly, but significantly higher BMI scores compared with those of students with regular menstruation (Fig. 3A), while those with premenstrual symptoms did not show significant differences in BMI compared to that in those without symptoms (Fig. 3B).

\section{DISCUSSION AND CONCLUSION}

Pelvic pain, which is an integrated physical sense [9], is conducted through afferent pathways by the hypogastric sympathetic and parasympathetic nerves [10,11]. Primary dysmenorrhea is a common gynecological disorder in young women [12]. It begins within 6-12 months from menarche and is characterized by localized pain in the abdominal inferior quadrants. Dysmenorrhea is an important clinical sign for local inflammation in the pelvis. In young women, this symptom is frequently caused by hormonal disorders in ovarian function [1]. Under inadequate balance of ovarian sex steroid hormones, a high amount of prostaglandins is

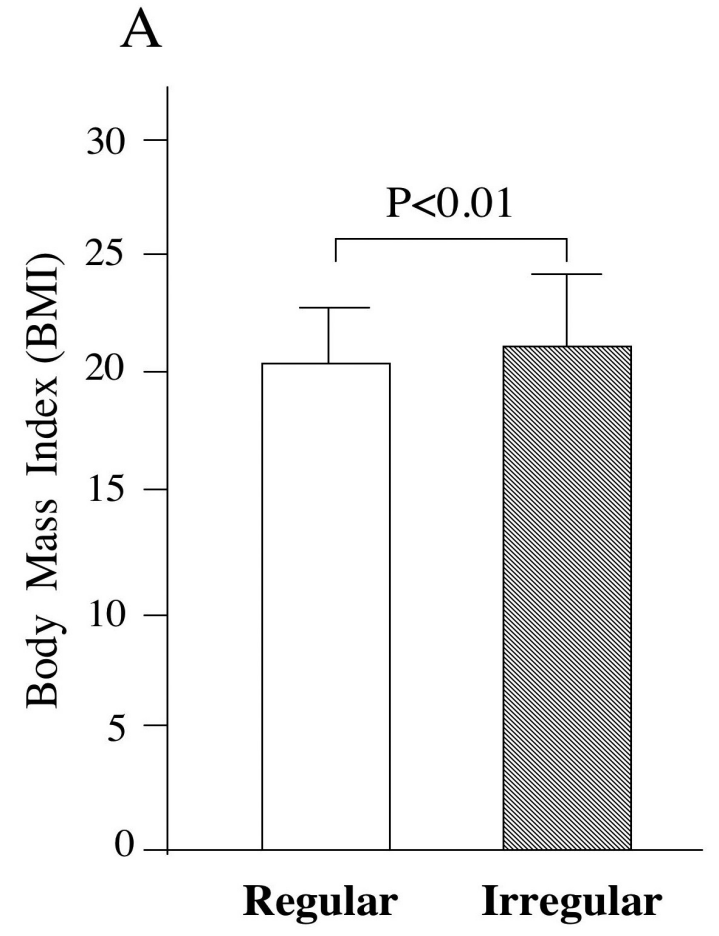

considered to be produced in the uterus during menstruation [13], causing excess contraction of uterine muscle and inducing functional dysmenorrhea [14]. To predict dysfunction in the hypothalamic-pituitary-ovarian axis, irregular menstruation is one of the reliable clinical symptoms.

To precisely evaluate the students with normal ovarian function, we strictly defined regular menstrual cycle as constant 26-32-day intervals in each menstrual cycle. Based on sequential information over a 5-year period between 2001 and 2005, the present study showed that students with irregular menstruation comprised one third of the participants, indicating that considerable numbers of young women have menstrual disorders. It was also shown that students with irregular menstruation frequently had a higher BMI. Recently, we observed that there is considerable discrepancy between BMI and self-recognition of adequate body weight in young students at Ashiya College and that those with relatively high BMI scores tended to undergo insufficient food intake and dietary habits despite being within normal BMI range [15]. It is well known that inappropriate dieting often induces amenorrhea in young women and may cause ovarian dysfunction during subsequent reproductive years $[16,17]$. A vegetarian low caloric diet is reported to lead to menstrual cycle disorders and a short luteal phase $[18,19]$. Thus, dysfunction in the hypothalamic- pituitary-ovarian axis can be induced by nutritional deficiency. It has been widely accepted that irregular menstruation in young students is partially caused by an inadequate nutritional state [20]. Recently, we found that young women who skip breakfast have a significantly higher degree of dysmenorrhea symptoms than those who eat breakfast [6]. It was also reported that intake of dietary fiber was inversely correlated with the menstrual pain in young Japanese women [21]. We also observed that diet during adolescence has long-lasting adverse effects

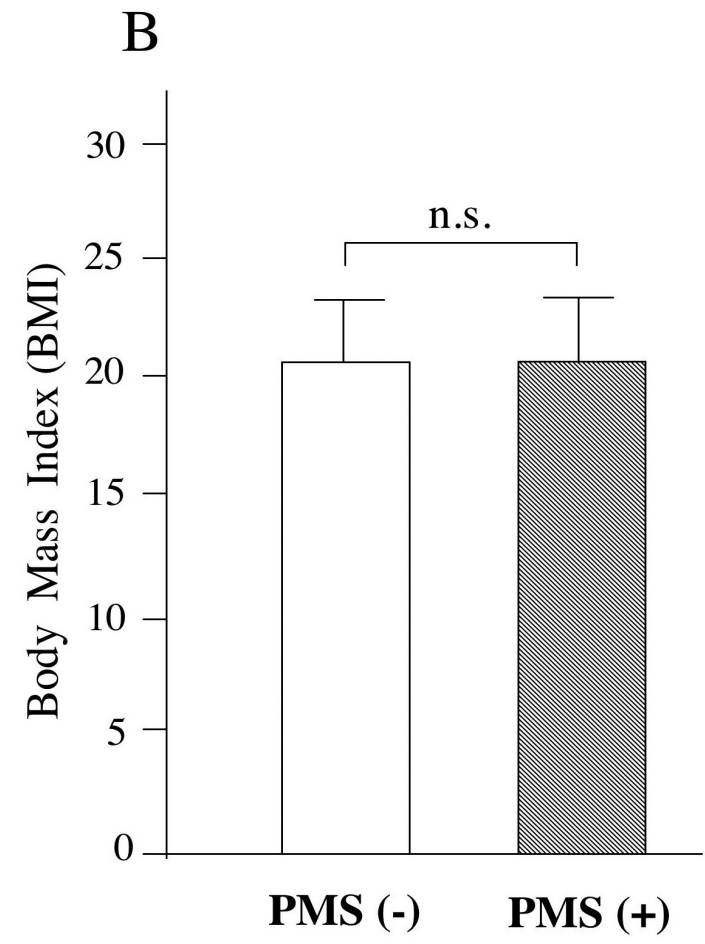

Fig. (3). Body mass index (BMI). BMI scores in students with irregular menses were significantly higher than that in those with regular menses (A), while those with premenstrual symptoms (PMS) did not show significant differences (B). The error bars show standard deviation. 
on dysmenorrhea [22] in young women. These findings suggest that an inadequate nutritional state has adverse effects on dysmenorrhea in young women [5]. In accordance with this study, students with irregular menses reported a higher intensity of dysmenorrhea (Fig. 1). Although serum levels of gonadotropins or ovarian sex steroid hormones were not examined, these findings suggest that functional disorders are involved in dysmenorrhea in young students with irregular menstruation.

Recently, increasing attention has been paid to the adverse effects of premenstrual syndrome on the quality of life in Japanese women. Although more than 100 symptoms and/or signs are considered to be involved in premenstrual syndrome, there is no specific laboratory test to precisely diagnose premenstrual syndrome. In this study, to obtain an accurate answer from the participants, we prepared a simple questionnaire and focused on 10 symptoms that mainly characterize premenstrual syndrome. Notably, this study demonstrated that almost half of the young women surveyed have premenstrual symptoms. Although the criteria employed in this study were not as strict as the criteria for clinical diagnosis of premenstrual syndrome, this high incidence is compatible with a recent report showing that about $60 \%$ of adolescence complain of premenstrual syndrome based on the criteria described in the Manual of Mental Disorders-IV $[23,24]$. In addition, this study showed that young Japanese women with premenstrual symptoms have a higher incidence of menstrual pains, suggesting a cross relationship between premenstrual syndrome and dysmenorrhea. Although functional dysmenorrhea frequently shows irregular menstruation, there was no significant relation between the incidences of premenstrual symptoms and irregular menstruation in these students, suggesting that dysmenorrhea with premenstrual symptoms is not necessary derived from functional disorders. Therefore, it should be noted that students with dysmenorrhea and premenstrual symptoms may have a risk for some organic diseases causing dysmenorrhea.

In conclusion, this study confirmed that considerable numbers of young women in Japan have irregular menstruation and premenstrual symptoms. Although this study did not show any significant relation between irregular menstruation and premenstrual symptoms, female students reporting premenstrual symptoms also complained of higher intensity of menstrual pains. From these findings, this study notably suggests that some organic diseases causing dysmenorrhea are latently proceeding in young Japanese women with premenstrual symptoms. Since dysmenorrhea and premenstrual syndrome may influence QOL in women not only in the present but also in the future, these results should be noted from the perspective of future reproductive function.

\section{REFERENCES}

[1] Deligeoroglou E. Dysmenorrhea. Ann NY Acad Sci 2000; 900 : 237-44.
[2] Pasquali R, Pelusi C, Genghini S, Cacciari M, Gambineri A. Obesity and reproductive disorders in women. Hum Reprod Update 2003; 9: 359-72.

[3] Kaneko S, Tamakoshi A, Ohno Y, Mizoue T, Yoshimura T. JACC Study Group. Menstrual and reproductive factors and the mortality risk of gastric cancer in Japanese menopausal females. Cancer Causes Control 2003; 14:53-9.

[4] Fauconnier A, Chapron C. Endometriosis and pelvic pain: epidemiological evidence of the relationship and implications. Hum Reprod Update 2005; 11: 595-606.

[5] Fujiwara T, Nakata R. Current problems of food intake in young women in Japan: their influence on female reproductive function. Reprod Med Biol 2004; 3: 107-14.

[6] Smith S, Schiff I. The premenstrual syndrome; Diagnosis and management. Fertil Steril 1989; 52: 527-43.

[7] Cerin A, Collins A, Landgren BM, Eneroth P. Hormonal and biochemical profiles of premenstrual syndrome. Acta Obstet Gynecol Scand 1993; 72: 337-43.

[8] Fujiwara T. Skipping breakfast is associated with dysmenorrhea in young women in Japan. Int J Food Sci Nutr 2003; 54: 505-9.

[9] Carpenter SE. Psychosocial menstrual disorders: stress, exercise and diet's effect on the menstrual cycle. Curr Opin Obstet Gynecol 1994; 6: 536-9.

[10] Renaer M, Vertommen H, Nijs P, Wagemans L, Van Hemelrijck T. Psychological aspects of chronic pelvic pain in women. Am J Obstet Gynecol 1979; 134: 75-80.

[11] Alonso C, Coe CL. Disruptions of social relationships accentuate the association between emotional distress and menstrual pain in young women. Health Psychol 2001; 20:411-41.

[12] Kennedy S. Primary dysmenorrhea. The Lancet 1997; 349: 1116.

[13] Lundstrom V, Green K. Endogenous levels of prostaglandin F2 and its main metabolites in plasma and endometrium of normal and dysmenorrheic women. Am J Obstet Gynecol 1978;130:640-646.

[14] Dawood MY. Dysmenorrhea and prostaglandins: Pharmacological and therapeutic consideration. Drugs 1981; 22: 42.

[15] Fujiwara T. The discrepancy between BMI and self-recognition of adequate body weight may cause insufficient food intake and habits in young women in Japan. Bulletin of Ashiya College 2005; 27: 7580 .

[16] Branca F. Physical activity, diet and skeletal health. Public Health Nutr 1999; 2: 391-6.

[17] Koebnick C, Strassner C, Hoffmann I, Leitzmann C. Consequences of a long-term raw food diet on body weight and menstruation: results of a questionnaire survey. Ann Nutr Metab 1999; 43: 69-79.

[18] Lloyd T, Schaeffer JM, Walker MA, Demers LM. Urinary hormonal concentrations and spinal bone densities of premenopausal vegetarian and nonvegetarian women. Am J Clin Nutr 1991;54:1005-10.

[19] Pedersen AB, Bartholomew MJ, Dolence LA, Aljadir LP, Netteburg KL, Lloyd T. Menstrual differences due to vegetarian and nonvegetarian diets. Am J Clin Nutr 1991; 53: 879-85.

[20] Montero P, Bernis C, Fernandes V, Castro S. Influence of body mass index and slimming habits on menstrual pain and cycle irregularity. J Biosoc Sci 1996;28:315-23.

[21] Nagata C, Hirokawa K, Shimizu N, Shimizu H. Associations of menstrual pain with intakes of soy, fat and dietary fiber in Japanese women. Eur J Clin Nutr 2005; 59: 88-92.

[22] Fujiwara T. Diet during adolescence is a trigger for subsequent development of dysmenorrhea in young women. Int J Food Sci Nutr 2007; 58: 437-44.

[23] Derman O, Kanbur NÖ. Tokur TE, Kutluk T. Premenstrual syndrome and associated symptoms in adolescence girls. Eur J Obstet Gynecol Reprod Boil 2004; 116: 201-6.

[24] American Psychiatric Association. Diagnostic and statistical manual of mental disorders. 4th ed. Washington, DC: American Psychiatric Association; 1994: 714-8. 